\title{
An audit of upper gastrointestinal endoscopy performed on patients at Prince Mshiyeni Memorial Hospital in Durban, KwaZulu-Natal
}

\author{
VC Ntola, ${ }^{1}$ TG Pillay, ${ }^{1}$ S Ramklass, ${ }^{2}$ W Sibanda ${ }^{3}$ \\ ${ }^{1}$ Department of General Surgery, Nelson R. Mandela School of Medicine, University of KwaZulu-Natal, South Africa \\ ${ }^{2}$ School of Clinical Medicine, University of KwaZulu-Natal, South Africa \\ ${ }^{3}$ School of Nursing and Public Health, University of KwaZulu-Natal, South Africa
}

Corresponding author: VC Ntola (vuyolwe@yahoo.com)

\begin{abstract}
Background: Resource constraints have resulted in upper gastrointestinal endoscopy (UGE) being deferred where possible. However, delayed investigation is costly and leads to disease progression. This study audits the UGE done at a single institution. It was motivated by the observation that public hospitals often experience an acute shortage of endoscopes, which are prone to frequent breaks and service delivery is further compromised by an increased workload.

Methods: This was a retrospective observational descriptive study of patients aged 20 to 45 years who had undergone gastrointestinal endoscopy (UGE) at Prince Mshiyeni Memorial Hospital (PMMH) in KwaZulu-Natal, during the period January 2015 to December 2015. One hundred and ninety-four patients' charts and UGE reports were reviewed. Data were analysed using SPSS Statistics version 24. The level of significance was set at $\mathrm{p}<0.05$. Variables were expressed as mean \pm standard deviation or medians (interquartile range IQR) as appropriate. Mean \pm standard deviation was compared using the Student's t-test. Proportions and categorical variables were compared using the Pearson's chi-square test or Fisher's exact test as appropriate. An ethical approval was obtained from the University of KwaZulu-Natal BREC (BE 447/17) and the KwaZulu-Natal Department of Health Ethics Committee.

Results: Epigastric pain was found to be the most common indication for UGE, with a total of $112(57.7 \%)$ out of total of 194 patients, followed by upper gastrointestinal bleeding (UGIB) (42) (21.6\%). Amongst patients presenting with epigastric pain, only $12(10.7 \%)$ patients could be confirmed that they had received acid suppression therapy prior to the testing. In the age group 20-25 years, there was a highest number of patients presenting with corrosive substance ingestion, 11 (25.0\%). The commonest finding was gastritis in 99 patients $(51.0 \%)$, followed by normal findings in $50(25.7 \%)$ patients.

Conclusions: Epigastric pain was the most common indication and gastritis was the predominant finding.

Keywords: Upper gastrointestinal endoscopy; Epigastric pain; Gastritis
\end{abstract}

S Afr J Surg 2019;57(3)

http://dx.doi.org/10.17159/2078-5151/2019/v57n3a2858

\section{Background}

Upper gastrointestinal endoscopy UGE is performed widely for the diagnosis and treatment of oesophageal, gastric and duodenal disorders. ${ }^{1}$ The shortage of endoscopes, frequent breaks and increased workload in the public hospitals has prompted the authors to conduct this study.

It is estimated that between $1 \%$ and $2 \%$ of patients with dyspepsia are associated with gastric cancer. ${ }^{2}$ To avoid missing gastric cancer, most guidelines recommend that patients over 45 years old should undergo UGE for uninvestigated dyspepsia. ${ }^{2}$ In countries where the gastric cancer is prevalent, optimal age threshold for endoscopy in patients with uninvestigated dyspepsia remains controversial. ${ }^{2}$ Dyspepsia is defined as chronic or recurrent central upper abdominal pain or discomfort to the upper (UGIT). ${ }^{3}$ In this study, it is referred to as epigastric pain. Resource constraints have resulted in endoscopy being deferred if possible. However, up to $70 \%$ of all patients with persistent dyspeptic symptoms have UGE at some stage, highlighting the costs and disease progression of delayed investigation. ${ }^{4}$ In a primary health care setting, $72.5 \%$ of referrals were appropriate compared to $27.5 \%$ of inappropriate referrals using the criteria from the American Society of Gastrointestinal Endoscopy (ASGE) as a screening tool for appropriateness. ${ }^{5}$ In a study of dyspeptic patients, only $21.0 \%$ of the referrals were appropriate in patients aged 45 years and less, and in $25.3 \%$ of those older than 45 years of age. ${ }^{6}$ ASGE optimum cut-off age for UGE 
may not hold true in regions with high prevalence of gastric and oesophageal cancers. ${ }^{7}$ It further concluded that more than $18.3 \%$ of patients with carcinoma would have been missed if the cut-off age of 45 years for endoscopy was followed as per the Western guidelines. ${ }^{7}$ An empirical treatment in the form of acid suppression could be recommended for those dyspeptics below the age of 35 years, provided they have no alarm symptoms, and an endoscopy can be performed after 4-6 weeks of presentation. ${ }^{7}$ Patients with heartburn at age 50 years or less do not require UGE. ${ }^{8}$ If the UGE is performed for screening on younger patients for trivial indications, it will increase the burden on the health service. ${ }^{8}$ It is known that GIT symptomatology is nonspecific and poorly correlated with organic pathology seen on UGE. This is responsible for normal findings in some patients who undergo this procedure. ${ }^{9}$

The aim of this study is to describe the findings of UGE in patients 45 years and younger. The study will further evaluate the appropriateness of the referrals in a resource-constrained setting. In resource constrained environment, this is an important study to assist clinicians in selecting appropriate patients for this procedure.

\section{Methods}

This was a retrospective observational descriptive study that used patients' chart reviews. Patients between 20 and 45 years of age seen at PMMH in Kwazulu-Natal, during the period January 2015 to December 2015 for UGE were purposively selected for investigation. The following data variables were collected for each patient: age (years), gender, indications, prior acid suppression use and endoscopic findings. Deidentified data was collected from patient records, entered into an Excel spreadsheet and stored on a password protected computer. In this study, continuous variables, such as patient ages were expressed as mean \pm standard deviation or medians (interquartile range IQR) as appropriate and compared using Student's t-test or Wilcoxon-Mann-Whitney test as appropriate. Categorical variables were summarised using proportions and compared using Pearson's chi-square test or Fisher's exact test as appropriate. All analyses were performed using IBM SPSS Statistics for Windows, version 25 (IBM Corp., Armonk, NY, USA). The level of significance was set at $p<0.05$. In this study, continuous variables, such as patient ages were expressed as mean \pm standard deviation or medians (interquartile range IQR) as appropriate and compared using Student's t-test or Wilcoxon-Mann-Whitney test as appropriate. Categorical variables were summarised using proportions and compared using the Pearson's chisquare test or Fisher's exact test as appropriate. All analyses were performed using IBM SPSS Statistics for Windows, version 25 (IBM Corp., Armonk, N.Y., USA). The level of significance was set at $\mathrm{p}<0.05$.

\section{Results}

Records of 194 patients who underwent UGE were found and included. Age was found to be non-normal distributed using Shapiro-Wilk's test for normality, $\mathrm{p}<0.001$. Therefore, non-parametric measure of central tendency, the median (interquartile range IQR), was reported instead of the mean (SD). The median age was 32 years (IQR13). There were more females than males, $\mathrm{p}=0.0008$. (Table 1)

\begin{tabular}{lcc} 
Table 1 Demographics & & \\
\hline Variables & $\mathbf{N}$ & $\mathbf{\%}(\mathbf{9 5 \%} \mathbf{C I})$ \\
\hline Gender & & \\
\hline Male & 73 & $37.6(30.8-44.4)$ \\
Female & 121 & $62.4(55.6-69.2)$ \\
Total & $\mathbf{1 9 4}$ & $\mathbf{1 0 0 . 0}$ \\
Age (Years) & & \\
\hline $20-25$ & 44 & $22.7(16.8-28.6)$ \\
$26-30$ & 38 & $19.6(14.0-25.2)$ \\
$31-35$ & 38 & $19.6(14.0-25.2)$ \\
$36-40$ & 48 & $24.7(18.6-30.8)$ \\
$41-45$ & 26 & $13.4(8.6-18.2)$ \\
Total & $\mathbf{1 9 4}$ & $\mathbf{1 0 0}$
\end{tabular}

\section{Indications for the UGE}

The commonest UGE finding was gastritis in 99 (51.0\%) patients and of these, $72(72.7 \%)$ had gastritis only and the remainder $27(27.0 \%)$ had gastritis associated with other findings. The second common finding was normal UGE findings $50(25.8 \%)$. Only $5(2.6 \%)$ patients were found to have an ulcer, two males and three females. Gastrooesophageal reflux disease (GORD) was not common, with only $2(1.0 \%)$ patients presenting with dysphagia. Anaemia work-up was also uncommon in both male $2(1.0 \%)$ and females $4(2.1 \%)$. (Table2)

\begin{tabular}{lccc} 
Table 2 UGE Indications & & & \\
\hline Variables & $\mathbf{N}$ & $\mathbf{\%}$ & $\mathbf{9 5 \%}$ CI \\
\hline Abdominal pain & 1 & 0.5 & $0.49-1.49$ \\
Anaemia work up & 6 & 3.1 & $0.66-5.54$ \\
B-cell stomach lymphoma & 2 & 1.0 & $0.4-2.4$ \\
Chronic gastroenteritis & 1 & 0.5 & $0.49-1.49$ \\
Corrosive ingestion & 12 & 6.2 & $2.81-9.59$ \\
Dysphagia & 2 & 1.0 & $0.4-2.4$ \\
Epigastric pain & 112 & 57.7 & $50.75-64.65$ \\
Odynophagia & 7 & 3.6 & $0.98-6.22$ \\
Persistent vomiting & 1 & 0.5 & $0.49-1.49$ \\
Per rectal (PR) bleed & 3 & 1.5 & $0.21-3.21$ \\
Portal hypertension & 3 & 1.5 & $0.21-3.21$ \\
UGIB & 42 & 21.6 & $15.36-26.84$ \\
Nil & 2 & 1.0 & $0.4-2.4$ \\
Total & $\mathbf{1 9 4}$ & $\mathbf{1 0 0 . 0}$ & \\
\end{tabular}

*Fisher's exact test, \#Chi-squared test 


\section{UGE Findings}

The commonest UGE finding was gastritis in 99 (51.0\%) patients, and of these $72(72.7 \%)$ had gastritis only and the remainder $27(27.0 \%)$ had gastritis associated with other findings. The second common finding was normal UGE findings $50(25.8 \%)$. Only $5(2.6 \%)$ patients were found to have an ulcer, two males and three females. Gastrooesophageal reflux disease (GORD) was not common, with only $2(1.0 \%)$ patients found with this condition. The total number of patients with oesophageal candidiasis was 19 $(9.8 \%)$ and for oesophagitis was 9 (4.6\%). There was only $1(0.5 \%)$ patient with achalasia and was in age group 31-35.

There were $42(21.6 \%)$ UGIB in terms of the gender 20 (47.6\%) females and $22(52.4 \%)$ had UGIB. The majority of patients with UGIB had gastritis 16 (38.1\%).

The association between UGE findings and epigastric pain was evaluated using a Chi-square test, and a statistically significant association was found between epigastric pain and gastritis with $\mathrm{p}<0.05$. (Table 3 )

\section{Table 3: UGE findings on patients with epigastric pain}

\begin{tabular}{lc}
\hline UGE findings & $\begin{array}{c}\text { No. of } \\
\text { cases }\end{array}$ \\
\hline Gastritis & 46 \\
Gastritis \& hiatus hernia & 7 \\
Gastritis, hiatus hernia \& duodenitis & 1 \\
Gastritis, hiatus hernia \& bile reflux & 1 \\
Gastritis \& oesophageal candidiasis & 3 \\
Gastritis, hiatus hernia \& oesophagitis & 1 \\
Gastritis \& oesophagitis & 1 \\
Gastritis \& duodenitis & 2 \\
Gastritis \& Forest Class III peptic ulcer & 1 \\
Hiatus hernia & 4 \\
Hiatus hernia \& oesophagitis & 3 \\
Hiatus hernia, Forest Class III peptic \& duodenal ulcer & 1 \\
Oesophagitis & 3 \\
Oesophageal candidiasis & 5 \\
Gastro-oesophageal reflux disease (GORD) & 2 \\
Oesophageal varices & 1 \\
Duodenitis & $\mathbf{1 1 2}$ \\
Peptic ulcer & 26 \\
Normal & 2 \\
TOTAL & 2 \\
&
\end{tabular}

Most patients $112(57.7 \%)$ presented with epigastric pain as an indication for UGE.

Epigastric pain without alarm features is not an absolute indication for endoscopy, but endoscopy may facilitate the diagnosis of structural disorders in a small subset of patients. ${ }^{10}$ According to ASGE guidelines, alarm features are age $\geq 50$ years, family history of upper gastrointestinal malignancy in a first-degree relative, unintended weight loss, gastrointestinal bleeding or iron deficiency anaemia, dysphagia, odynophagia, persistent vomiting, abnormal imaging suggesting organic disease. ${ }^{10}$

In a study conducted in Ghana, it was found that amongst the patients who required UGE, $80 \%$ presented with dyspepsia not associated with any other symptom, and in $50 \%$ of these patients the endoscopic findings were normal, ${ }^{11}$ a similar finding that was observed in our study where $50 \%$ of patients with epigastric pain had normal UGE findings. With respect to the above-mentioned Ghana study, the second commonest finding was gastritis, and this was the same finding in our study.

In our study, 12 (10.7\%) patients received acid suppression therapy, in the form of proton pump inhibitors (PPIs), prior to undergoing UGE. In other charts, it was not stated whether the patients received any acid suppression therapy. There are several possible explanations for this observation, including the fact that some of the patients had not undergone any assessment by an endoscopist prior to the procedure and were referred from the primary health centre, a fact that might lead to unclear UGE indications or patients with no indications for UGE to end up undergoing this procedure. Another possible explanation is the observation that up to $70 \%$ of all patients with persistent dyspeptic symptoms have UGE at some stage. ${ }^{4}$ It is also possible that some patients could have received acid suppression therapy, but the information was missing. The information regarding the acid suppression therapy prior to the scope is important, because failure to respond in 4 to 6 weeks justifies the performance of UGE. ${ }^{8}$ PPIs aid in the healing of ulcers, hence a patient on PPIs is not expected to be having peptic ulcers. ${ }^{12}$

In total, there were $42(21.7 \%)$ patients with UGIB, and of these $20(47.6 \%)$ were females and $22(52.4 \%)$ males. None of these patients with UGIB required endoscopic intervention for bleeding. In the above-mentioned study in Ghana, UGIB was the indication for endoscopy in $53(66.2 \%)$ patients. PUD was the primary finding in $16(30.2 \%)$ of them, while in $19(35.8 \%)$ patients the findings were normal. ${ }^{12}$ In our study, amongst the 42 patients who had UGIB 16 (38.1\%) had gastritis. Oesophageal varices, gastric ulcer and duodenal ulcer were found in 5 (11.9\%), 5 (11.9\%) and $2(4.8 \%)$ patients respectively. ${ }^{12}$

Between the ages 20-25, we observed that a high number of patients $11(5.6 \%)$ had undergone UGE for corrosive ingestion, while only $1(0.5 \%)$ patient out of this age group had corrosive ingestion. A further study of these research findings can shed more light.

\section{Conclusion}

Epigastric pain was the most common indication of UGE and gastritis was the predominant finding. The study revealed that if the UGE could be limited to patients who will benefit from the procedure, a significant number of patients could avoid this procedure and that could contribute to the effectiveness of the health system and durability of the gastroscope. Based on our research findings, we propose that at PMMH there should 
be revisions to the policy and criteria of booking the UGE. Our recommendations are as follows:

1. Patients below the age of 45 with dyspepsia for the first time should receive a trial of PPIs, and only referred for further evaluation by specialist if they do not respond after six weeks. Patients with alarm symptoms (loss of weight, UGIB, family history of GIT cancer, dysphagia and anaemia) should be directly be booked for UGE.

2. Surgical departments should devise a list of indications to be considered for elective UGE and ensure that doctors at primary healthcare clinics have access to this information.

3. For patients with UGIB, scores that predict the severity of bleeding such as Glasgow-Blatchford score should be used to select the patients for UGE.

4. Patients with buffered substance ingestion, such as Jik, to be only observed instead of performing UGE.

The change of policy will result in a reduction of patients undergoing UGE for unclear indications. Due to high numbers of patients undergoing the UGE, the gastroscope has frequent breaks. These breaks delay the diagnosis and management of patients with serious indications of UGE, such as elderly patients with dysphagia. If the change of policy could be adopted, this would lead to a reduction in workload burden in the study hospital.

\section{Competing interests}

The authors declare that they have no competing interests.

\section{Consent for publication}

Authors give consent for publication.

\section{Acknowledgements}

The authors wish to thank Sr Mceytwa, who assisted with data collection processes.

\section{Ethics approval and informed consent}

The study protocol was approved by the University of KwaZulu-Natal Biomedical Research Ethics Committee (BE443/17) and KZN DOH (KZ_201708_030). Permission to conduct the study was obtained from the Hospital Research Review Board.

\section{Limitations of the study}

Due to the retrospective nature of the study, the authors were not always able to determine data such as whether or not the patient had alarm features, for example, whether or not there was a family history of GIT malignancy. Some information on assessment of the patient prior and after the UGE could not be found, such as Rockall score for the patients who had UGIB. Authors had no details of the nature of the acoustic ingestions.

\section{REFERENCES}

1. Park WG, Shaheen NJ, Cohen J, et al. Quality indicators for EGD. Gastrointest Endosc. 2015;81(1):17-30.

2. Liou JM, Lin JT, Wang HP, et al. The optimal age threshold for screening upper endoscopy for uninvestigated dyspepsia in Taiwan, an area with a higher prevalence of gastric cancer in young adults. Gastrointest Endosc. 2005;61(7):819-25.

3. Jemilohun AC, Fadare JO. Dyspepsia management in a resource poor setting. Ann Ib Postgrad. 2013;11(1) 2-6.

4. Axon ATR, Bell GD, Jones RH. Guidelines On Appropriate Indications For Upper Gastrointestinal Endoscopy. Br Med J. 1995;310(6983):853-6.

5. Al-Romaih WR, Al-Shehri AM. Appropriateness of Upper Gastrointestinal Endoscopy Referrals from Primary Health Care. Ann Saudi Med. 2006;26(3):224-7.

6. Adang RP, Vismans JF, Talmon JL, et al. Appropriateness of indications for diagnostic upper gastrointestinal endoscopy: association with relevant endoscopic disease. Gastrointest Endosc. 1995;42(5):390-7.

7. Sumathi B, Navaneethan U, Jayanthi V. Appropriateness of indications for diagnostic upper gastrointestinal endoscopy in India. Singapore Med J. 2008;49(12):970-6.

8. Shaheen NJ. Editorial: Should women with heartburn undergo screening upper endoscopy for prevention of cancer? Am J Gastroenterol. 2011;106(2):261-3.

9. Obayo S, Muzoora C, Ocama P, et al. Upper gastrointestinal diseases in patients for endoscopy in South-Western Uganda. Afr Health Sci. 2015;15(3):95.

10. Shaukat A, Wang A, Acosata RD, et al. The role of endoscopy in dyspepsia. Gastrointest Endosc 2015; 82(2).

11. Gyedu A, Yorke J. Upper gastrointestinal endoscopy in the patient population of Kumasi, Ghana: Indications and findings. Pan Afr Med J. 2014; 18:327 doi:10.11604/ pamj.2014.18.327.4806

12. Daniel S. Strand, Daejin et al. 25 Years of Proton Pump Inhibitors: A Comprehensive Review. Gut Liver. 2017; 11(1): 27-37. 